\title{
Molecular Understanding of How Interfacial Structure Impacts on Open- Circuit Voltage of Highly Crystalline Polymer Solar Cells
}

Tomohiro Fukuhara,$^{\dagger}$ Koshi Yamazaki,$^{\dagger}$ Takuto Hidani,$^{\dagger}$ Masahiko Saito, ${ }^{\ddagger}$ Yasunari Tamai,$^{\dagger} \S$ Itaru Osaka, ${ }^{*}$, Hideo Ohkita ${ }^{*} \dagger$

$\dagger$ Department of Polymer Chemistry, Graduate School of Engineering, Kyoto University, Katsura, Nishikyo-ku, Kyoto 615-8510, Japan

†Department of Applied Chemistry, Graduate School of Engineering, Hiroshima University, 1-41 Kagamiyama, Higashi-Hiroshima, Hiroshima 739-8527, Japan

§PRESTO, Japan Science and Technology Agency (JST), 4-1-8 Honcho, Kawaguchi, Saitama 332-0012, Japan 


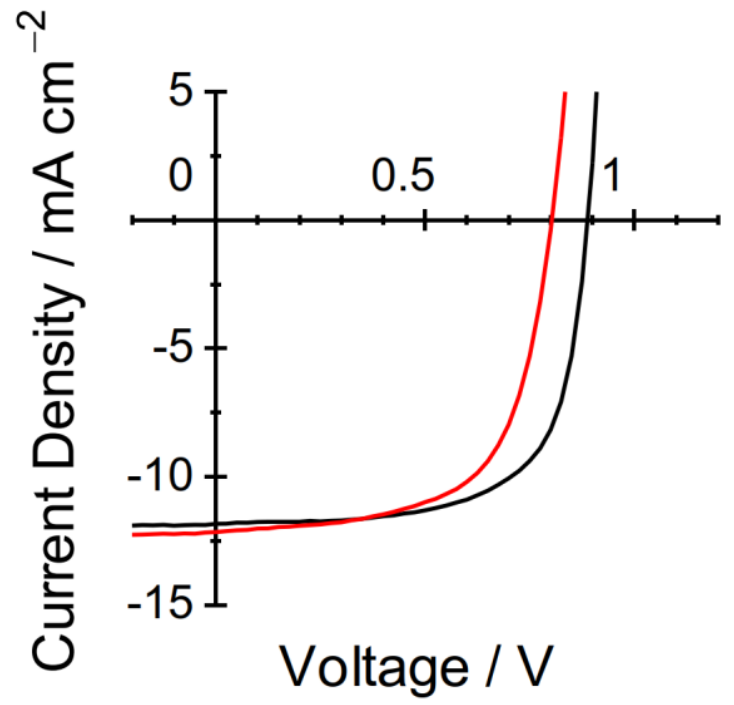

Figure S1. $J-V$ curves of PTzBT-BOHD/PCBM (black line) and PTzBT-12OD/PCBM (red line) solar cells with an inverted structure of ITO/ZnO/polymer:PCBM/MoO $/ \mathrm{Ag}$.

Table S1. Photovoltaic parameters of PTzBT/PCBM solar cells with an inverted structure.

\begin{tabular}{ccccc}
\hline Polymer & $J_{\mathrm{SC}} / \mathrm{mA} \mathrm{cm}^{-2}$ & $V_{\mathrm{OC}} / \mathrm{V}$ & FF & PCE $/ \%$ \\
\hline PTzBT-BOHD & 11.8 & 0.89 & 0.67 & 7.08 \\
PTzBT-12OD & 12.2 & 0.80 & 0.63 & 6.16 \\
\hline
\end{tabular}




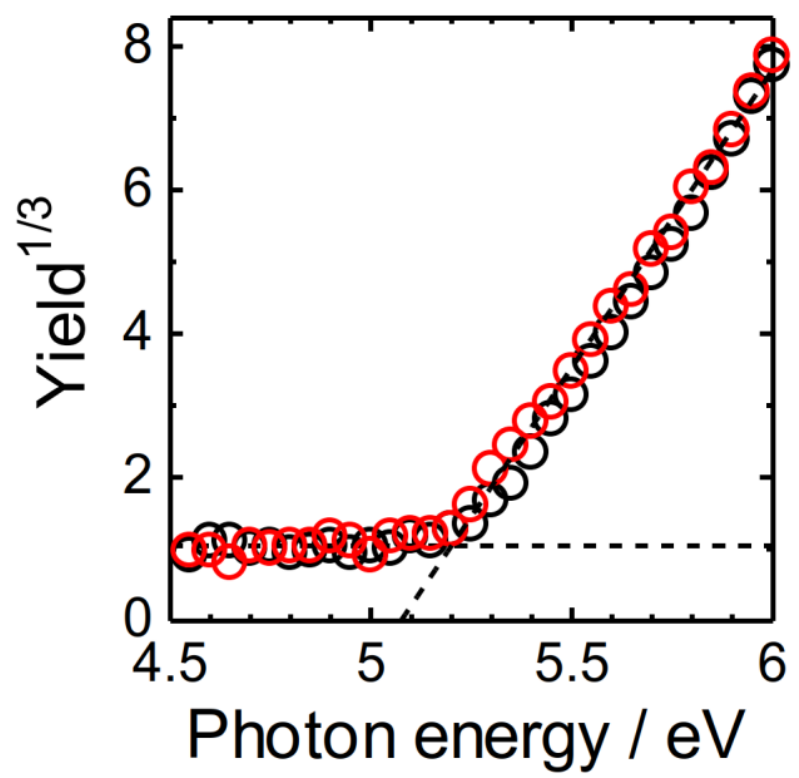

Figure S2. Photoelectron yield spectra of PTzBT-BOHD (black) and PTzBT-12OD (red) thin films.

\section{Electroluminescence spectra.}

As shown in Figure S3, the electroluminescence (EL) spectra were reproduced with the sum of two components using Equation 2 in the manuscript. The two sets of obtained parameters are shown in Table S2. As shown in Figure 3, the component with higher $E_{\mathrm{CT}}\left(E_{\mathrm{CT} 2}\right)$ was compatible with the fitting of EQE spectra. In addition, the temperature dependence of reduced EL spectra are shown in Figure S4. Whereas the component with lower $E_{\mathrm{CT}}\left(E_{\mathrm{CT} 1}\right)$ was almost independent of temperature, the $E_{\text {CT2 }}$ decreased with decreasing temperature. The linear extrapolation of $E_{\mathrm{CT} 2}$ at $0 \mathrm{~K}$ coincided that of $V_{\mathrm{OC}}$, as shown in Figure 4 . On the basis of the above results, we assigned the component with $E_{\mathrm{CT} 2}$ as the luminescence of $\mathrm{CT}$ states. 
(a)

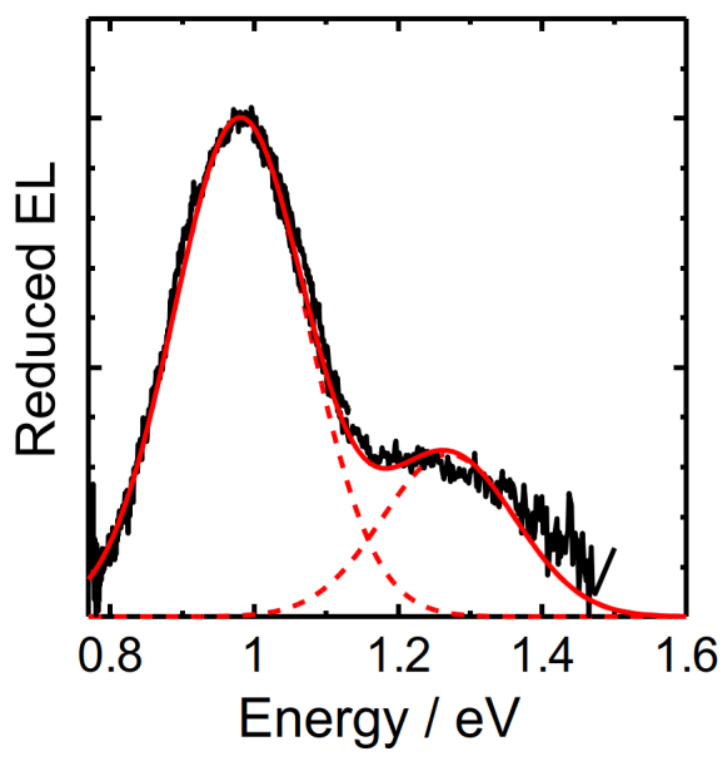

(b)

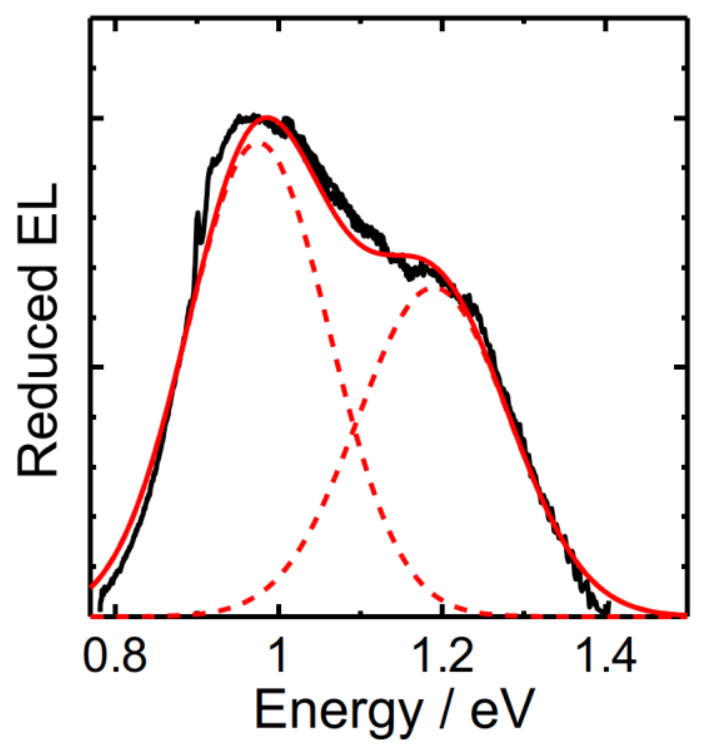

Figure S3. Reduced EL spectra (black solid lines) for (a) PTzBT-BOHD/PCBM and (b) PTzBT-12OD/PCBM solar cells. The red broken lines represent the fitting curves with Equation 2 in the manuscript, and the red solid lines represent the sum of the broken lines.

Table S2. Fitting parameters of EL spectra with two components.

\begin{tabular}{ccccc}
\hline Polymers & $E_{\mathrm{CT} 1} / \mathrm{eV}$ & $\lambda_{1} / \mathrm{eV}$ & $E_{\mathrm{CT} 2} / \mathrm{eV}$ & $\lambda_{2} / \mathrm{eV}$ \\
\hline PTzBT-BOHD & 1.15 & 0.17 & 1.44 & 0.17 \\
PTzBT-12OD & 1.13 & 0.15 & 1.36 & 0.17 \\
\hline
\end{tabular}


(a)

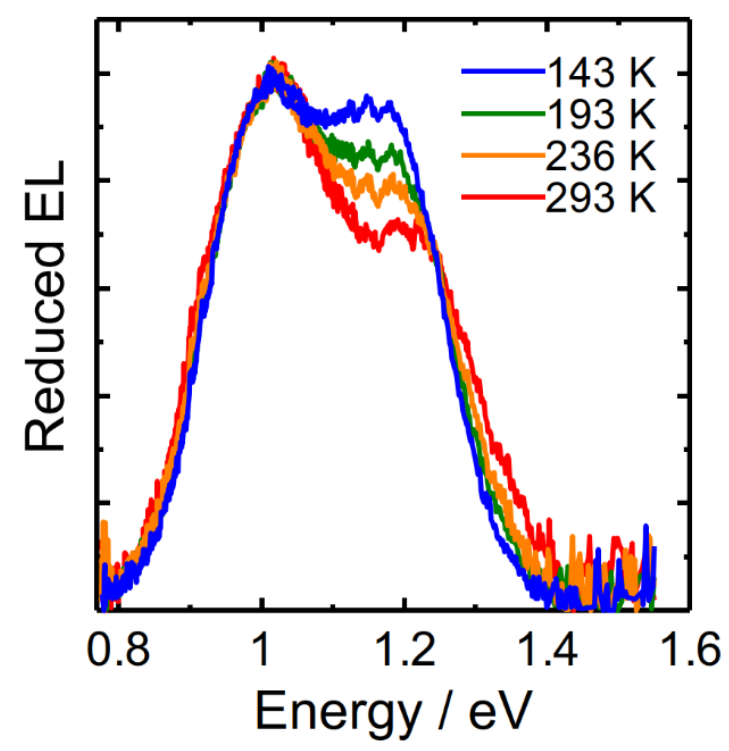

(b)

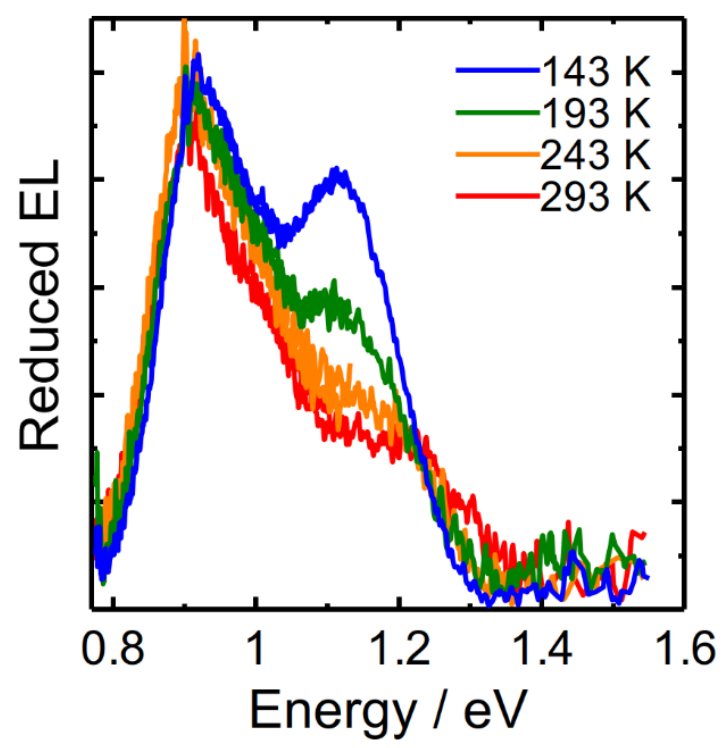

Figure S4. Temperature dependence of reduced EL spectra for (a) PTzBT-BOHD/PCBM and (b) PTzBT-12OD/PCBM solar cells.

(a)

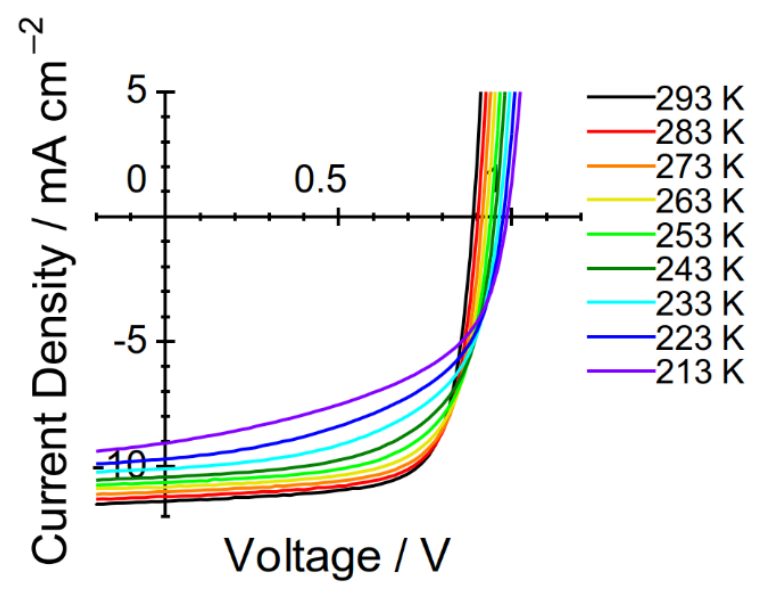

(b)

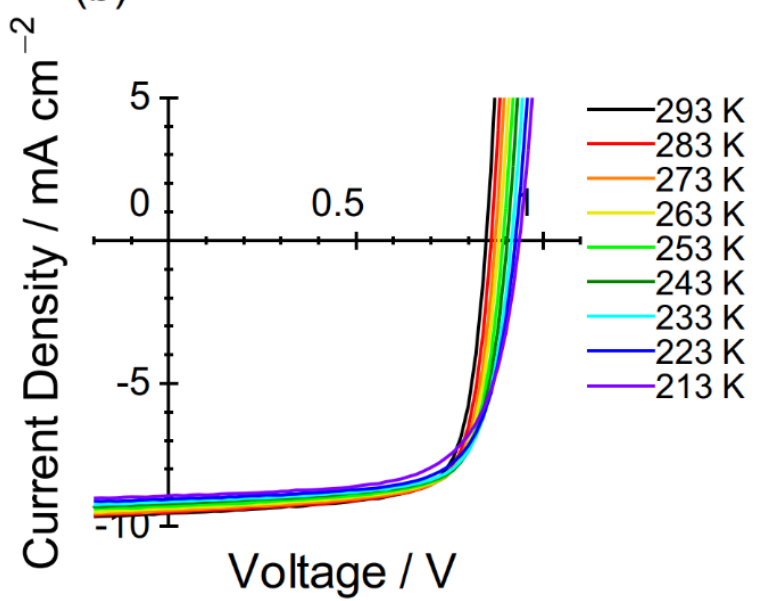

Figure S5. Temperature dependence of $J-V$ curves of (a) PTzBT-BOHD/PCBM and (b) PTzBT-12OD/PCBM solar cells. 


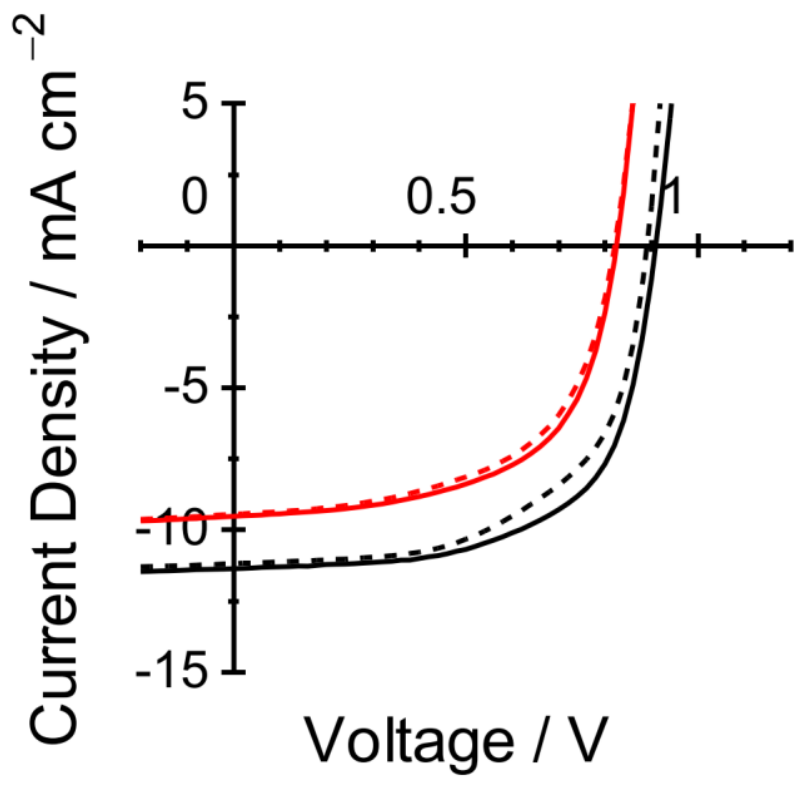

Figure S6. $J-V$ curves of (a) PTzBT-BOHD/PCBM (black) and (b) PTzBT-12OD/PCBM (red) solar cells before (solid lines) and after (broken lines) the temperature-dependent measurement. 


\section{Light intensity dependent $J-V$ measurement.}

Figures $\mathrm{S} 7 \mathrm{a}$ and $\mathrm{S} 7 \mathrm{~b}$ show the light intensity $(I)$ dependence of $J_{\mathrm{SC}}$. The $J_{\mathrm{SC}}$ was fitted by a power-law function $J_{\mathrm{SC}} \propto I^{\alpha}$. Figures S7c and S7d show $\log -\log$ plots of $V_{\mathrm{OC}}$ against $J_{\mathrm{SC}}$. The ideality factor $\left(n_{\text {id }}\right)$ was evaluated using equation S1.

$$
V_{\mathrm{OC}}=\frac{n_{\mathrm{id}} k_{\mathrm{B}} T}{q} \ln \left(\frac{J_{\mathrm{SC}}}{J_{0}}\right)
$$

As shown in Table S3, $\alpha$ and $n_{\text {id }}$ are similar for the two polymers. 
(a)
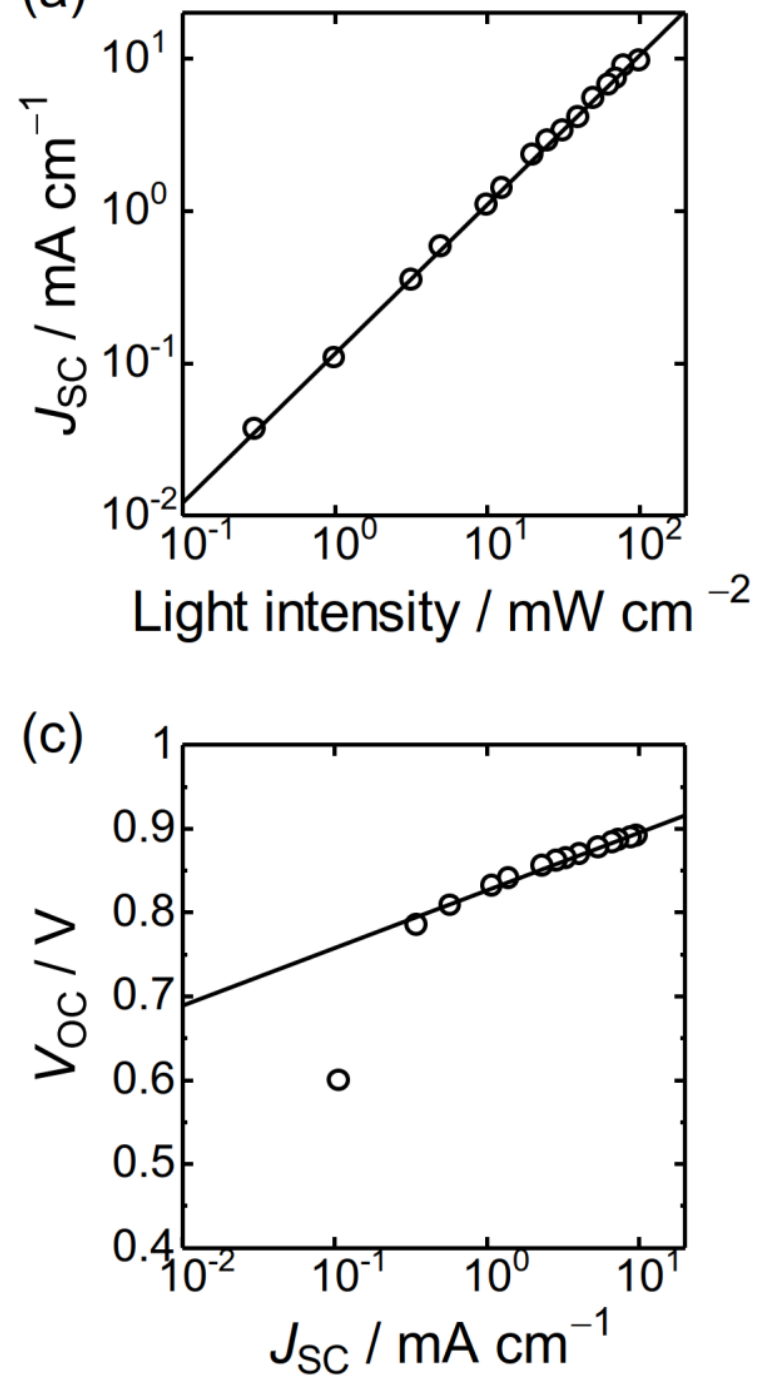

(b)

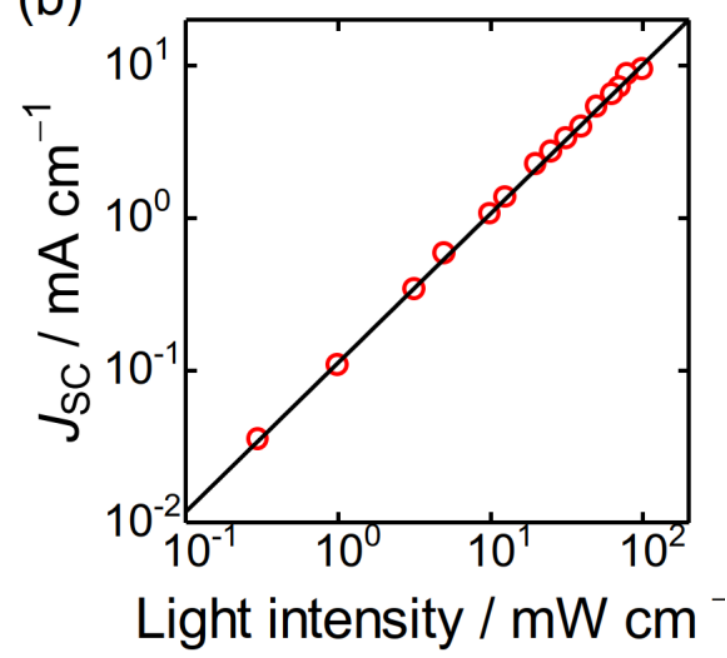

(d)

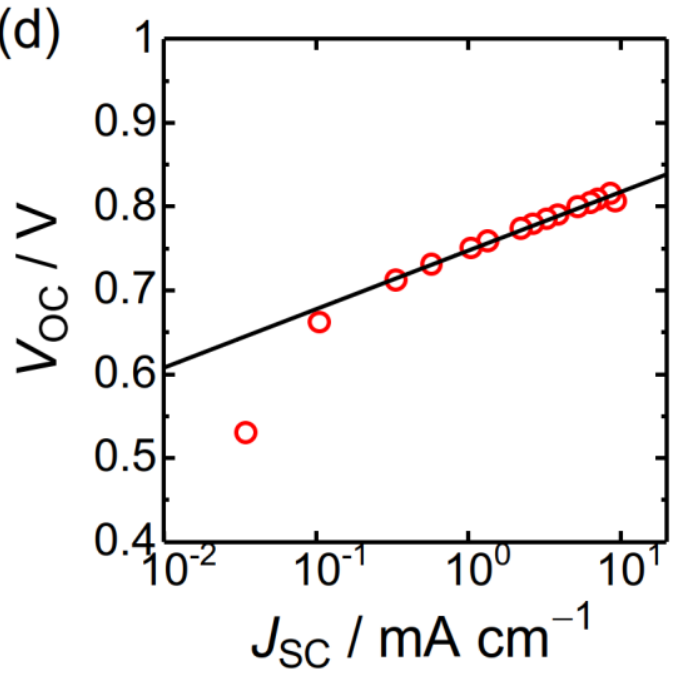

Figure S7. Log-log plots of $J_{\mathrm{SC}}$ against the light intensity for (a) PTzBT-BOHD/PCBM and (b) PTzBT-12OD/PCBM solar cells. The solid line represents a fitting line by a power-law function $J_{\mathrm{SC}} \propto I^{\alpha}$. Log-log plots of $V_{\mathrm{OC}}$ against $J_{\mathrm{SC}}$ for (c) PTzBT-BOHD/PCBM and (d) PTzBT12OD/PCBM. 
Table S3. Parameters obtained by light intensity dependent $J-V$ measurement.

\begin{tabular}{ccc}
\hline Polymer & $\alpha$ & $n_{\text {id }}$ \\
\hline PTzBT-BOHD & 0.98 & 1.20 \\
PTzBT-12OD & 0.98 & 1.21 \\
\hline
\end{tabular}

\section{Transient photovoltage (TPV) and transient photocurrent (TPC) measurement.}

Bimolecular recombination parameters were evaluated by transient photovoltage (TPV) and transient photocurrent (TPC) measurement. Figure S8a shows charge carrier lifetime $\tau_{n}$ plotted against charge carrier density $n$. Langevin reduction factor $\zeta$ defined as the ratio of bimolecular recombination rate constant $k_{\text {rec }}$ to Langevin recombination rate constant $k_{\mathrm{L}}$ using the following equations.

$$
\begin{gathered}
k_{\text {rec }}=\frac{1}{\tau_{n} n} \\
k_{\mathrm{L}}=\frac{q \mu}{\varepsilon_{\mathrm{r}} \varepsilon_{0}} \\
\zeta=\frac{k_{\mathrm{rec}}}{k_{\mathrm{L}}}
\end{gathered}
$$

where $\mu$ is the charge carrier mobility, $\varepsilon_{\mathrm{r}}$ is the relative dielectric constant, and $\varepsilon_{0}$ is the vacuum permittivity. As shown in Figure $\mathrm{S} 8 \mathrm{~b}, \zeta$ is on the order of $10^{-2}$ for both blend systems, indicating that the bimolecular recombination is significantly suppressed. As described in the main text, there is a difference for both systems in the interfacial energy cascade structure, which is beneficial for suppressed recombination. The reason why the recombination dynamics was similar despite the different cascade structure would be that the energy cascade was sufficient in 
both systems. The energy difference of HOMO levels between crystalline and mixed phase evaluated by CV is 290 and $220 \mathrm{meV}$ for PTzBT-BOHD and PTzBT-12OD, respectively, which would be adequately large.

(a)

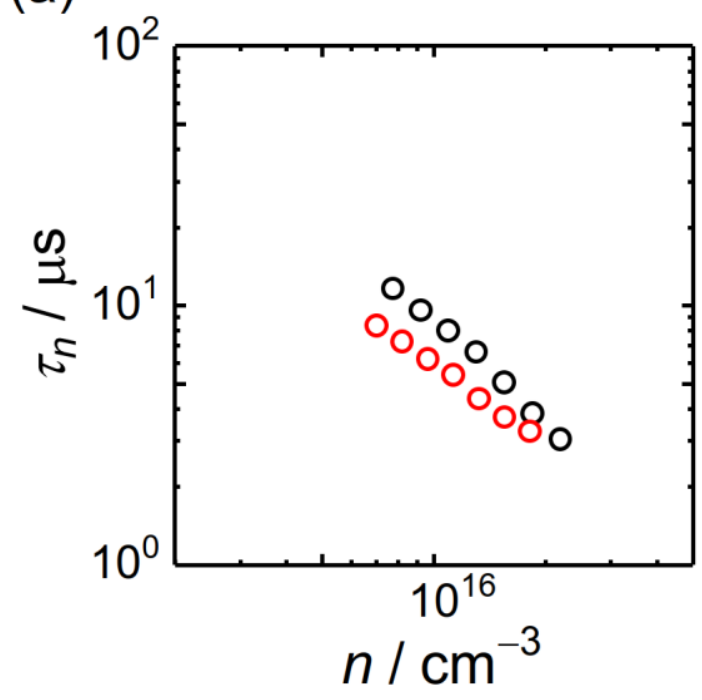

(b)

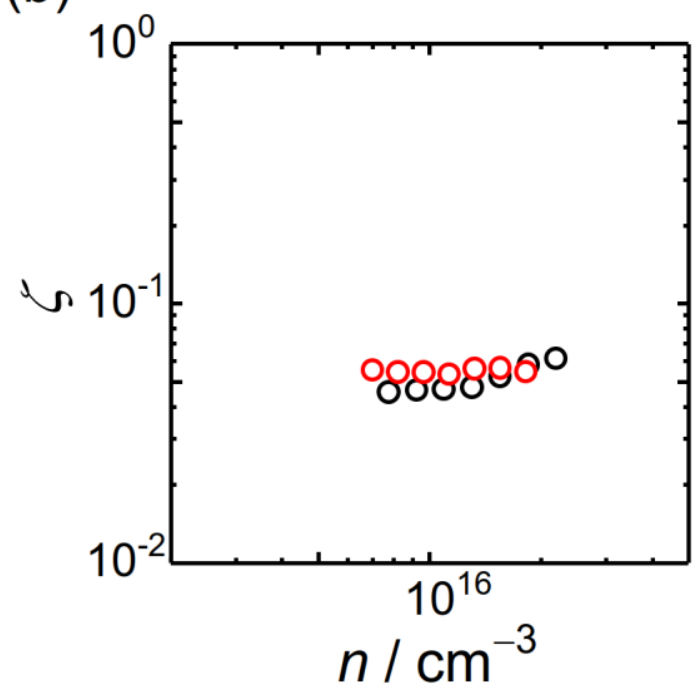

Figure S8. $\log -\log$ plots of (a) charge carrier lifetime $\tau_{n}$ and (b) Langevin reduction factor $\zeta$ against charge carrier density $n$ for PTzBT-BOHD/PCBM (black) and PTzBT-12OD/PCBM (red) solar cells. 


\section{CV measurement.}

Figure S9 shows the cyclic voltammograms of ferrocene. The average voltage of anode and cathode peaks was evaluated to be $0.087 \mathrm{~V}$, independent on scan rate. The energy on upper Xaxis voltammograms of the samples was determined by adjusting this voltage to be $-4.8 \mathrm{eV}$. Figure S11 shows the scan rate dependence of cyclic voltammograms of the blend films. The peak positions were independent on scan rate. Since the onset of the peaks were broader for higher scan rate, we evaluated $E_{\mathrm{H}}$ from voltammograms obtained by slow scan. Figure S12 shows the comparison of voltammograms of polymer neat and polymer/PCBM blend films. The peak positions were not altered by blending PCBM, suggesting that the morphology of polymer in crystalline and amorphous phases did not change significantly.

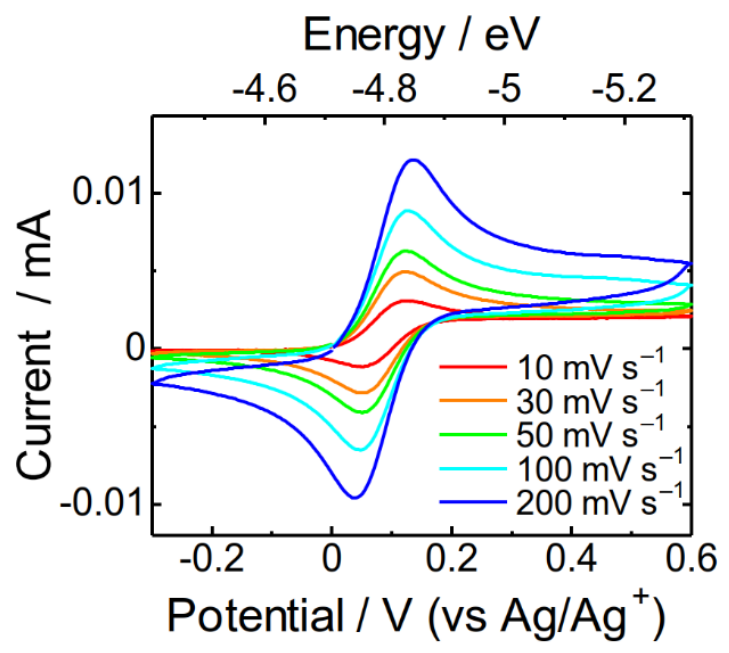

Figure S9. Cyclic voltammograms of ferrocene. 
(a)

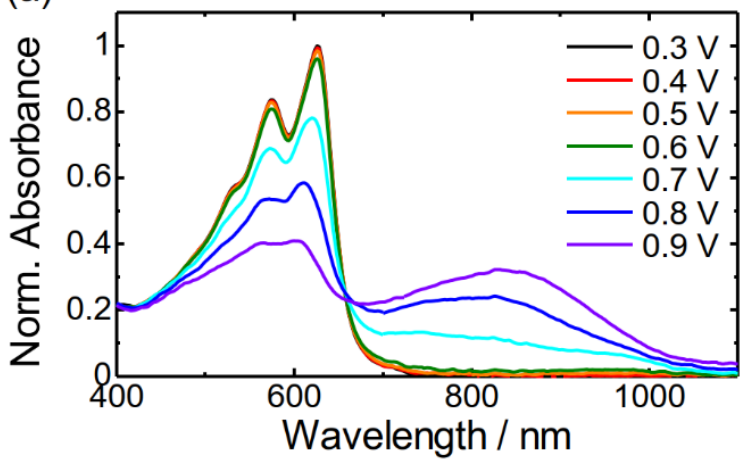

(b)

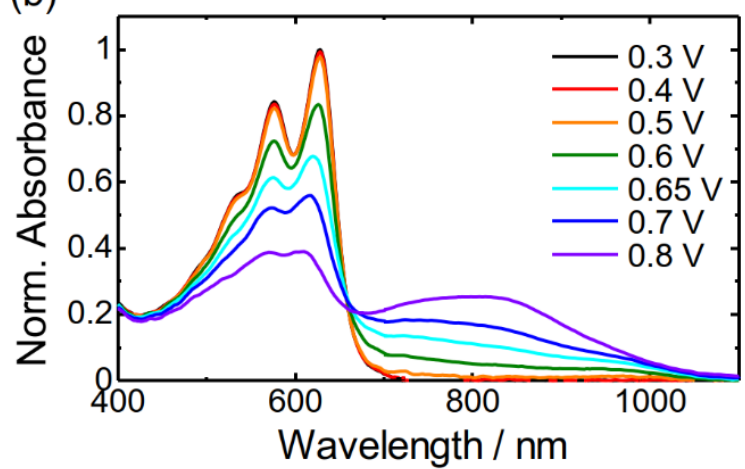

Figure S10. Absorption spectra observed simultaneously during the CV measurement: (a) PTzBT-BOHD/PCBM and (b) PTzBT-12OD/PCBM blend films.

(a)

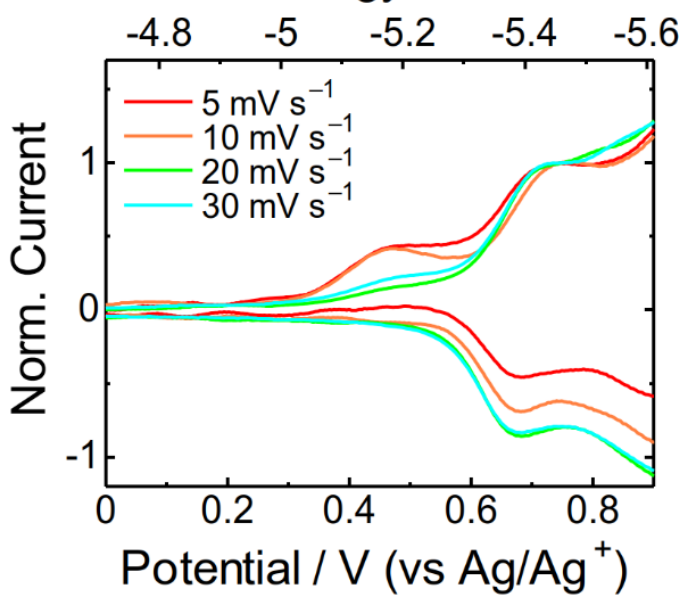

(b) Energy / eV

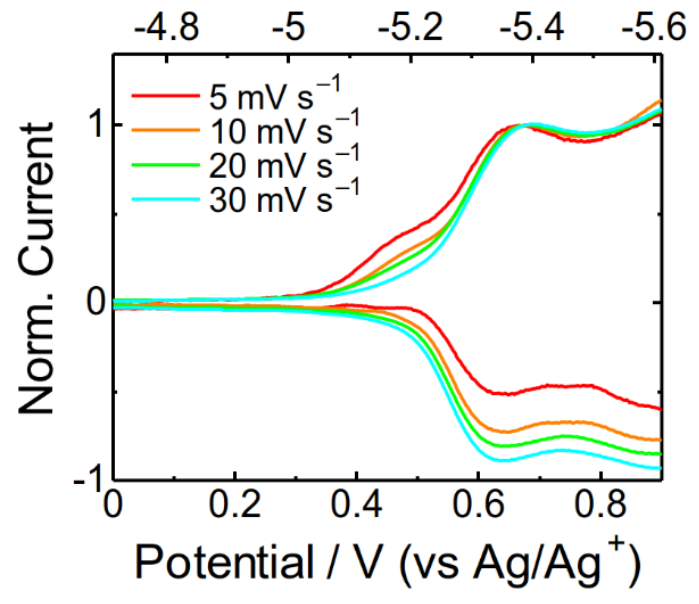

Figure S11. Scan rate dependence of cyclic voltammograms of the (a) PTzBT-BOHD/PCBM and (b) PTzBT-12OD/PCBM blend films normalized at the second peaks on the forward scan. 
(a)

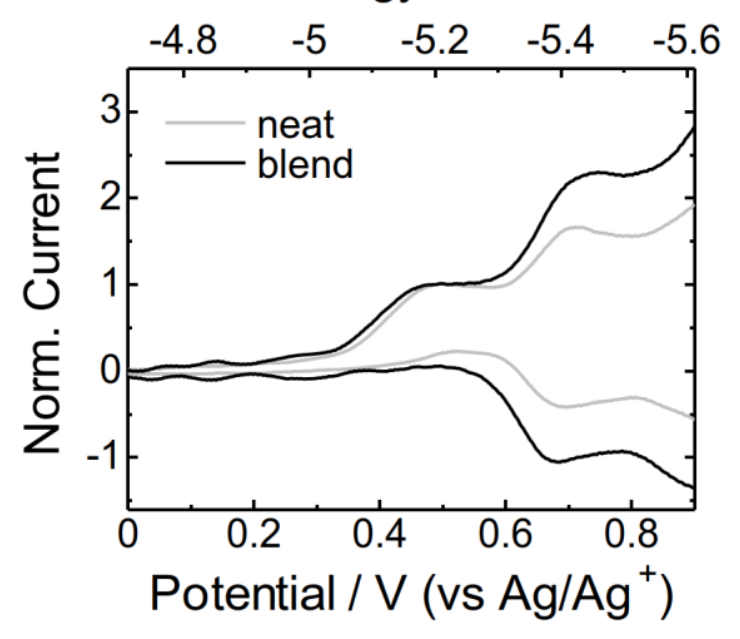

(b) Energy / eV

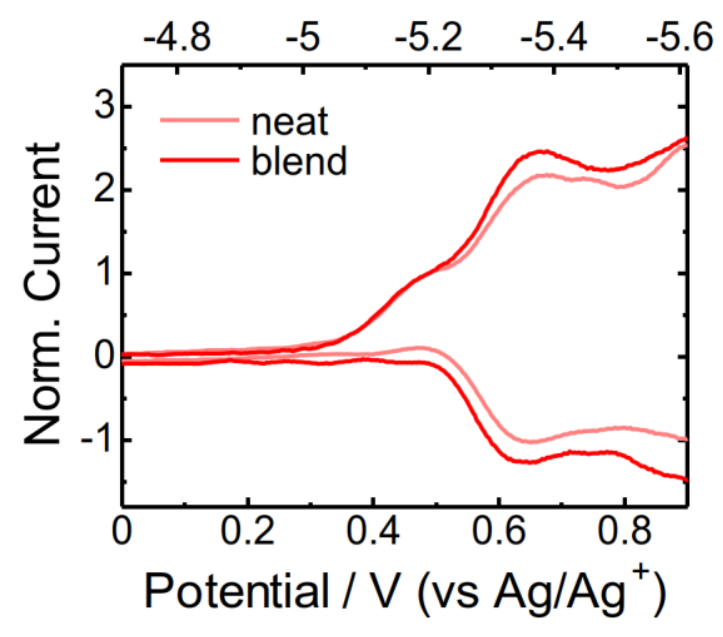

Figure S12. Cyclic voltammograms of polymer neat and polymer/PCBM blend films of (a) PTzBT-BOHD and (b) PTzBT-12OD normalized at the first peaks on the forward scan.

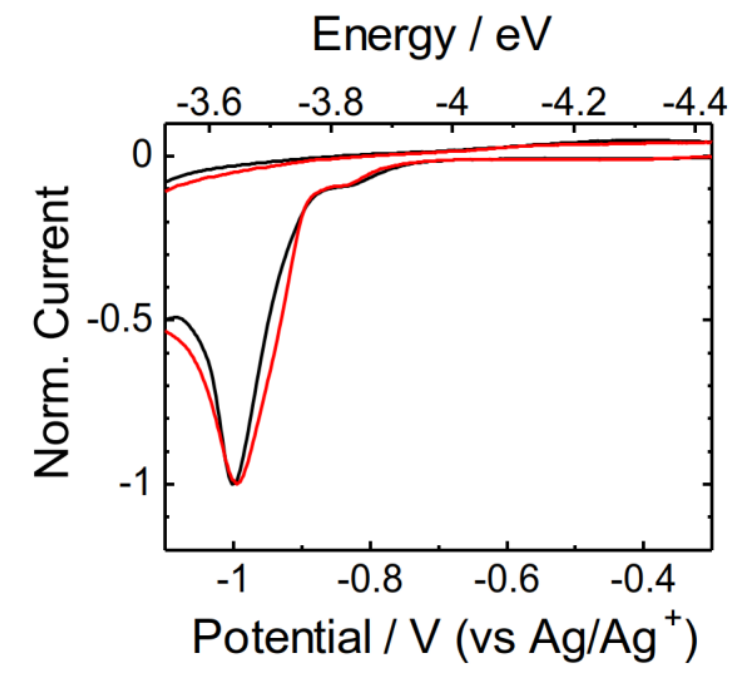

Figure S13. Cyclic voltammograms of polymer/PCBM blend films of PTzBT-BOHD (black) and PTzBT-12OD (red) normalized at the peak on the forward scan. 
(a)

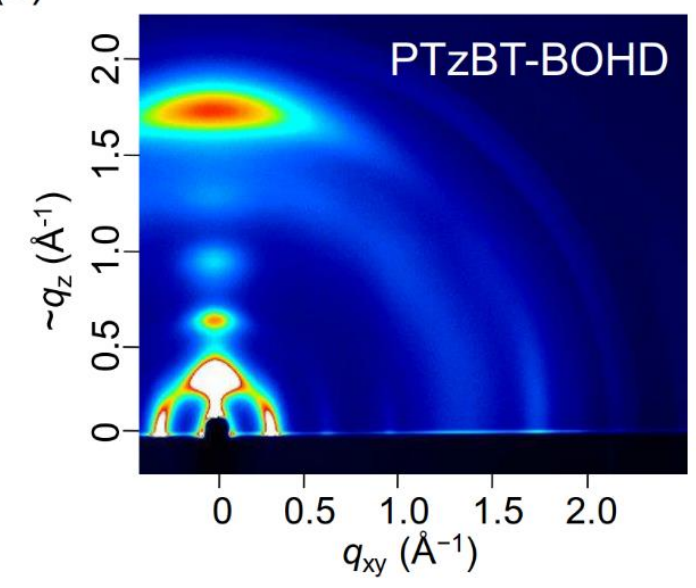

(c)

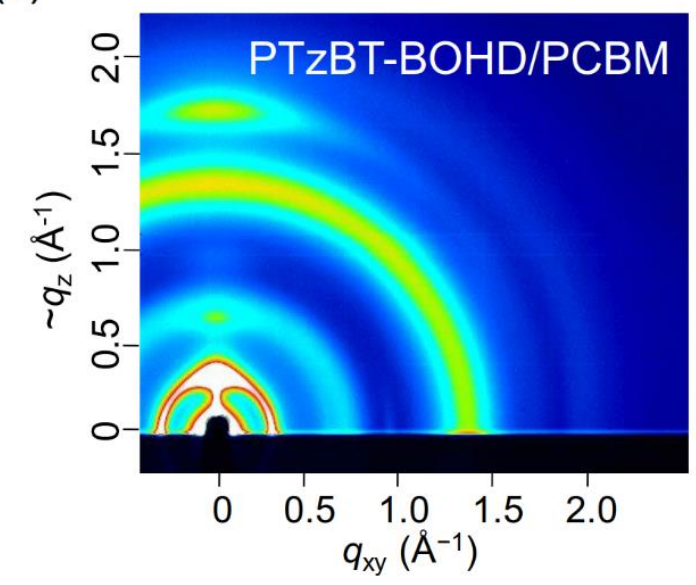

(b)

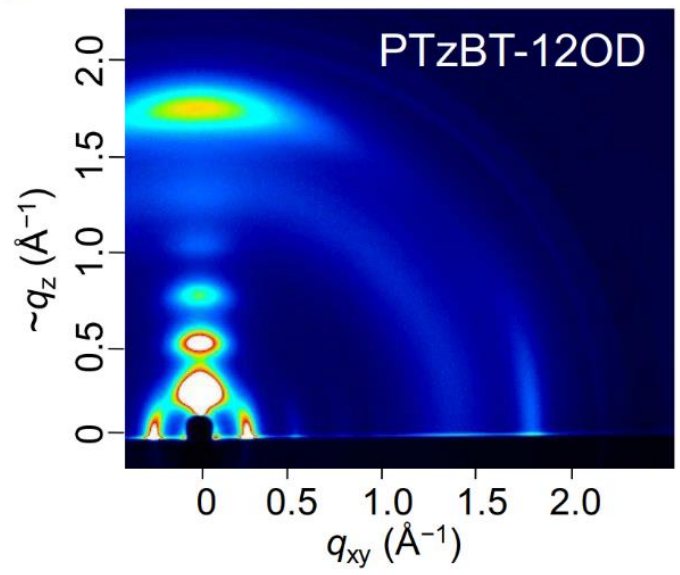

(d)

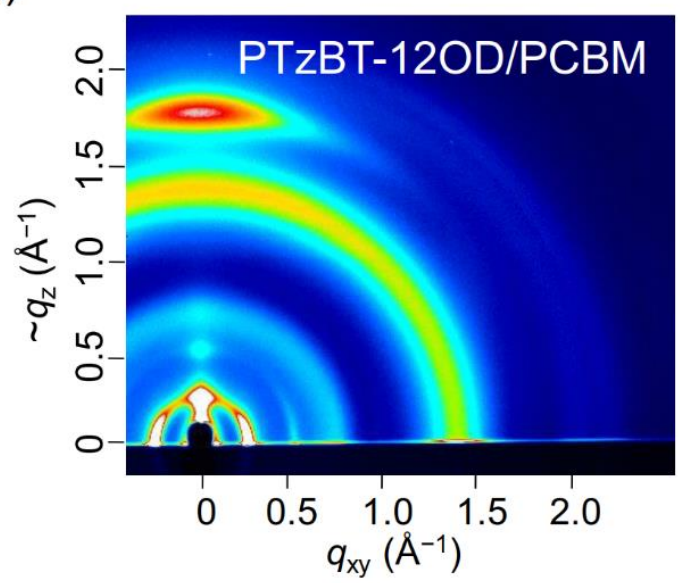

Figure S14. Two-dimensional grazing incidence wide-angle X-ray diffraction patterns. (a) PTzBT-BOHD neat film, (b) PTzBT-12OD neat film, (c) PTzBT-BOHD/PCBM blend film, and (d) PTzBT-12OD/PCBM blend film.

Table S4. $\pi$-Stacking and lamellar distance of the polymers.

\begin{tabular}{ccccc}
\hline \multirow{2}{*}{ Polymer } & \multicolumn{2}{c}{$\pi$-stacking distance $/ \AA$} & \multicolumn{2}{c}{ Lamellar distance / } \\
\cline { 2 - 5 } & Neat & Blend & Neat & Blend \\
\hline PTzBT-BOHD & 3.48 & 3.48 & 18 & 18 \\
PTzBT-12OD & 3.54 & 3.49 & 22 & 23 \\
\hline
\end{tabular}




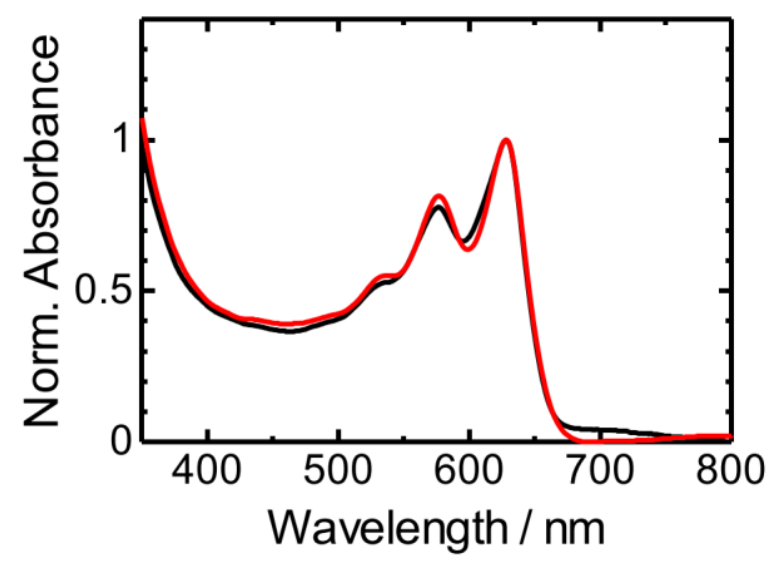

Figure S15. Absorption spectra of polymer/PCBM blend films of PTzBT-BOHD (black) and PTzBT-12OD (red).

(a)

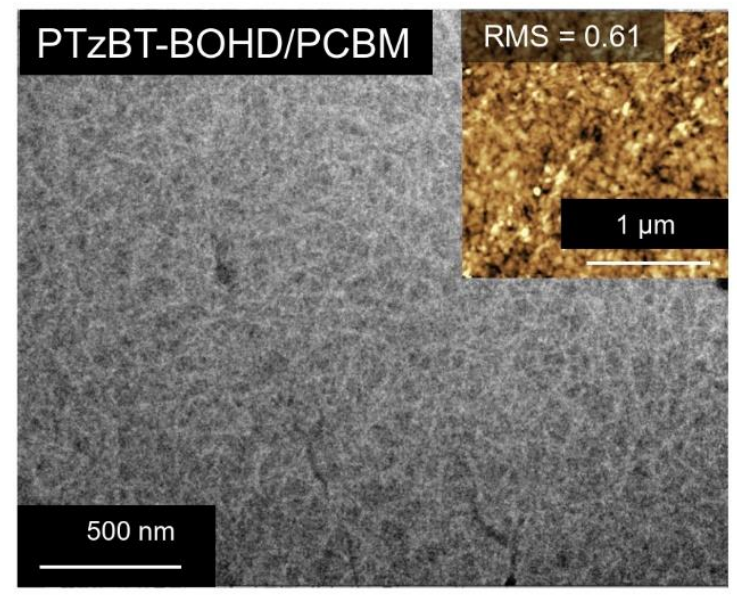

(b)

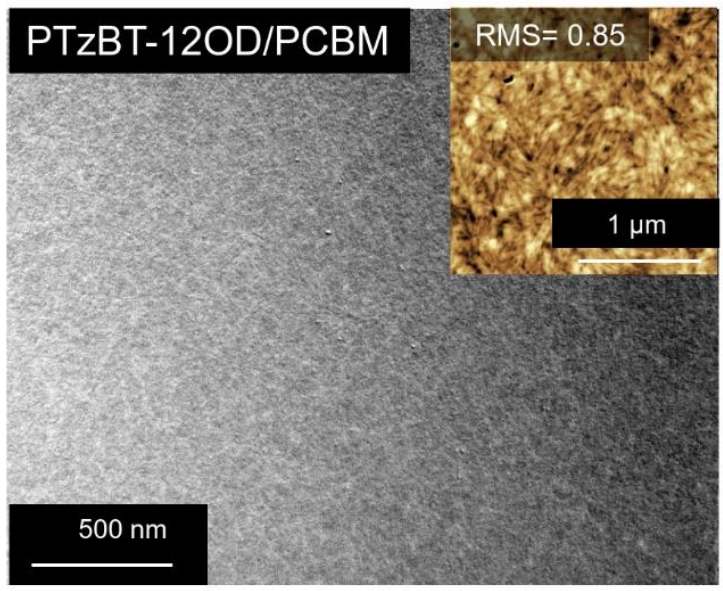

Figure S16. TEM and AFM (inset) images of the blend films. (a) PTzBT-BOHD/PCBM and (b) PTzBT-12OD/PCBM. 


\section{DFT calculation.}

Table S5 shows the $E_{\mathrm{H}}$ of a trimer of the polymers determined by DFT calculation (CAMB3LYP/6-31G(d,p)). The difference in $E_{\mathrm{H}}$ of the optimized structures and hydrogen-substituted structures was 86 and $33 \mathrm{meV}$, respectively, which are both close to the difference for monomer unit. Therefore, we employed the result for monomers in the main text.

Table S5. $E_{\mathrm{H}}$ of a trimer of the polymers determined by DFT calculation.

\begin{tabular}{lcc}
\hline \multirow{2}{*}{ Polymer } & \multicolumn{2}{c}{$E_{\mathrm{H}} / \mathrm{eV}$} \\
\cline { 2 - 3 } & Alkyl $^{\mathrm{a})}$ & Hydrogen $^{\mathrm{b})}$ \\
\hline PTzBT-BOHD & -6.197 & -6.397 \\
PTzBT-12OD & -6.111 & -6.364 \\
\hline a) & $E_{\mathrm{H}}$ of the optimized structure of a trimer with the original alkyl side chains. ${ }^{\text {b) }} E_{\mathrm{H}}$ determined \\
by single point energy calculation of a trimer made by substituting hydrogen for the side chain of \\
the optimized molecule.
\end{tabular}

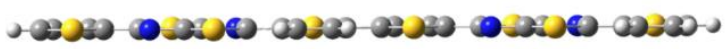

Figure S17. Side view of main chain geometry of monomer unit for PTzBT without side chains. 

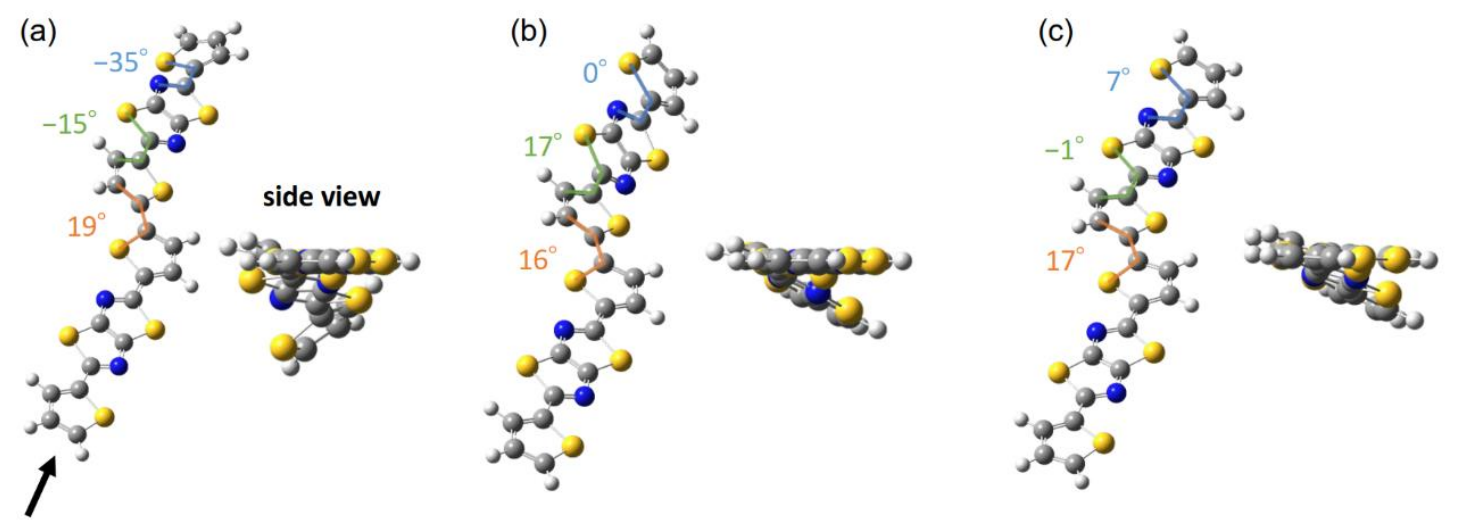

Figure S18. Monomer unit mainchain geometry of (a) PTzBT-12OD- $\alpha$, (b) PTzBT-12OD- $\beta$, and (c) PTzBT-12OD- $\gamma$. The dihedral angle between aromatic rings near the branched side chains and the side view of the mainchain from the viewpoint indicated by an arrow. The monomer units were optimized with a respective side chain and then the side chains were replaced with a hydrogen atom. 